\title{
Fast 3-D photoacoustic imaging in vivo with a high frequency ultrasound array toward clinical applications
}

Liang Song, Konstantin Maslov, Rachel Bitton, K. Kirk Shung, Lihong V. Wang

Liang Song, Konstantin Maslov, Rachel Bitton, K. Kirk Shung, Lihong V. Wang, "Fast 3-D photoacoustic imaging in vivo with a high frequency ultrasound array toward clinical applications," Proc. SPIE 7177, Photons Plus Ultrasound: Imaging and Sensing 2009, 71770G (24 February 2009); doi: $10.1117 / 12.809013$

SPIE. Event: SPIE BiOS, 2009, San Jose, California, United States 


\title{
Fast 3-D Photoacoustic Imaging in vivo with a High Frequency Ultrasound Array towards Clinical Applications
}

\author{
Liang Song ${ }^{1}$, Konstantin Maslov ${ }^{1}$, Rachel Bitton ${ }^{2}$, K. Kirk Shung ${ }^{2}$, and Lihong V. Wang ${ }^{1}$ \\ ${ }^{1}$ Optical Imaging Laboratory \\ Department of Biomedical Engineering \\ Washington University in St. Louis, St. Louis, Missouri 63130 \\ ${ }^{2}$ Department of Biomedical Engineering \\ University of Southern California, Los Angeles, California 90089
}

\begin{abstract}
We present an in vivo reflection-mode photoacoustic microscopy system that performs B-scan imaging at $50 \mathrm{~Hz}$ with realtime beamforming and 3-D imaging of $166 \mathrm{~B}$-scan frames at $1 \mathrm{~Hz}$ with post-beamforming. To our knowledge, this speed is currently the fastest in high frequency photoacoustic imaging. In addition, with a custom fiber based light delivery system, the imaging device is capable of performing handheld operation. Software for image processing and display with clinically user-friendly graphic user interface (GUI) is developed. The system has axial, lateral, and elevational resolutions of 25,70 , and $200 \mu \mathrm{m}$, respectively, and can image $3 \mathrm{~mm}$ deep in scattering biological tissue. Volumetric images of subcutaneous vasculature in murine are demonstrated in vivo. The system is anticipated to have potential clinical applications in skin melanoma detection due to its unique ability to image in realtime and to image anatomical sites inaccessible to other imaging systems.
\end{abstract}

Keywords: realtime photoacoustic imaging, three-dimensional photoacoustic microscopy, high frequency ultrasound array, clinical diagnosis, melanoma imaging, subcutaneous vasculature, small animal imaging

\section{INTRODUCTION}

In photoacoustic imaging, photo-induced ultrasonic waves are detected to reconstruct the distribution of optical absorption in biological tissue. As ultrasonic waves experience $2-3$ orders of magnitude less scattering in tissue than optical waves, photoacoustic imaging combines high ultrasonic resolution and excellent optical absorption contrast beyond the optical ballistic and quasiballistic regimes [1]. Recently, photoacoustic imaging has become one of the fastest growing imaging technologies in biomedicine due to its potential in many clinical and biomedical applications, such as skin melanoma detection [2-4], breast cancer diagnosis $[5,6]$, functional brain imaging $[7,8]$, and molecular imaging [9].

High imaging speed is highly desirable for decision-making in clinics and is essential for biomedical research involving dynamics. Photoacoustic imaging systems using a single element ultrasound transducer are limited in speed by mechanical scanning. To improve imaging speed, ultrasound arrays were employed for photoacoustic imaging [10,11]. For example, using a 7.5 MHz ultrasound array, Niederhauser et al. [10] obtained an imaging speed of $7.5 \mathrm{~B}$-scan-frames/s with $0.3-0.4 \mathrm{~mm}$ resolution. While ultrasound arrays of clinical diagnostic ultrasound frequencies (several $\mathrm{MHz}$ ) are commercially available, arrays of high frequencies $(>12 \mathrm{MHz})$ are still at the research stage (only in October, 2008, VisualSonics Inc. revealed the world's first high frequency ultrasound digital platform with array transducer technology—Vevo® 2100

Photons Plus Ultrasound: Imaging and Sensing 2009, edited by Alexander A. Oraevsky, Lihong V. Wang, Proc. of SPIE Vol. 7177, 71770G · C 2009 SPIE · CCC code: 1605-7422/09/\$18 · doi: 10.1117/12.809013 
Micro Imaging Ultrasound Platform). With a high frequency $(30 \mathrm{MHz})$ ultrasound array, we developed a photoacoustic microscopy system capable of realtime B-scan imaging in vivo with $\sim 25 \mu \mathrm{m}$ axial and $<100$ $\mu \mathrm{m}$ lateral resolutions $[12,13]$. Murine cardiovascular dynamics were visualized in vivo with the system [14].

Besides high imaging speed, 3-D imaging is also highly desirable. One advantage of 3-D imaging is to provide maximum amplitude projection (MAP) images in various orientations. Another advantage is the ability to view images interactively. The flexibility to rotate, scale, and view the region of interest from various perspectives can facilitate visualization.

We present a fast 3-D dark-field reflection-mode photoacoustic microscopy system with a $30-\mathrm{MHz}$ ultrasound linear array. This system performs realtime cross-sectional (B-scan) imaging at $50 \mathrm{~Hz}$ (faster than the $30-\mathrm{Hz}$ video rate) with realtime beamforming and 3-D imaging of $166 \mathrm{~B}$-scan frames at $1 \mathrm{~Hz}$ with post-beamforming. To enable dark-field reflection-mode photoacoustic imaging and handheld operation, we designed a novel fiber based light delivery system. The dark-field laser pulse illumination configuration was known to have advantages in suppressing undesirable photoacoustic signals from the superficial layers of the skin [2]. Three-dimensional photoacoustic images of the subcutaneous vasculature in rats were demonstrated in vivo, which agreed well with their ex vivo transmission optical microscopy counterparts. Our photoacoustic microscopy system is presented as a compact prototype for handheld operation and is anticipated to accelerate preclinical and clinical applications of photoacoustic imaging.

\section{METHODS}

A schematic of the system is shown in Fig. 1. Our system consists of a diode-pumped Q-switched $\mathrm{Nd}$ :YLF laser, a tunable dye laser, a 30-MHz ultrasound linear array, custom receive and control electronics, an 8-channel PCI data acquisition (DAQ) card, a multi-core PC, a custom designed light delivery system, and a motorized linear motion actuator.

\subsection{Optics and Light Delivery}

The tunable dye laser (Cobra, Sirah Laser-und Plasmatechnik GmbH, Germany), pumped by the Nd:YLF laser (INNOSLAB, Edgewave GmbH, Germany), was used as the radiation source. The Nd:YLF pump laser had a pulse duration of $7 \mathrm{~ns}$ and a pulse energy of $12 \mathrm{~mJ}$ at $523 \mathrm{~nm}$. The continuous optical pumping from the diode stacks in this Q-switched Nd:YLF laser provided the flexibility of external triggering on demand at rates up to $1 \mathrm{kHz}$ without compromising the pulse energy. This feature offered a significant advantage over flashlamp-pumped Q-switched lasers, which are typically maintained at a fixed low pulse repetition rate (e.g., $10 \mathrm{~Hz}$ ). Pyrromethene 597 laser dye was used to enable output of $\sim 2 \mathrm{~mJ}$ per pulse with a pulse width of $\sim 7 \mathrm{~ns}$ at $584 \mathrm{~nm}$ from the dye laser. This wavelength $(584 \mathrm{~nm})$, corresponding to an isosbestic point where oxy- and deoxy-hemoglobin have equal molar extinction coefficients, was used for our imaging experiments.

Proper delivery of laser light into biological tissue for photoacoustic excitation is crucial to achieving a high signal-to-noise ratio (SNR) in photoacoustic imaging. The light delivery system was designed to provide a compact photoacoustic imaging device with sufficient SNR and robust performance. The dye laser output was split into two beams by a 50:50 non-polarizing beamsplitter. The two beams were coupled into two 0.6-mm-core-diameter multimode optical fibers. The output light beams from the fibers were then focused by two cylindrical lenses and delivered to the skin surface. A water container with a window of low-density polyethylene (LDPE) film filled with de-ionized water was used for ultrasonic coupling. As a result, the skin surface was not contacted by the scanning probe during experiments and disturbance to the imaged subject was minimized. In total, $\sim 40 \%$ light energy of the laser output reached the surface of the film due to coupling and reflection losses. Because of finite fiber aperture, the final optical illumination patterns on the skin surface were thick-line shaped, as shown in Fig. 1 (inset A). The length and width of each illumination area were 8 and $4 \mathrm{~mm}$, respectively. 


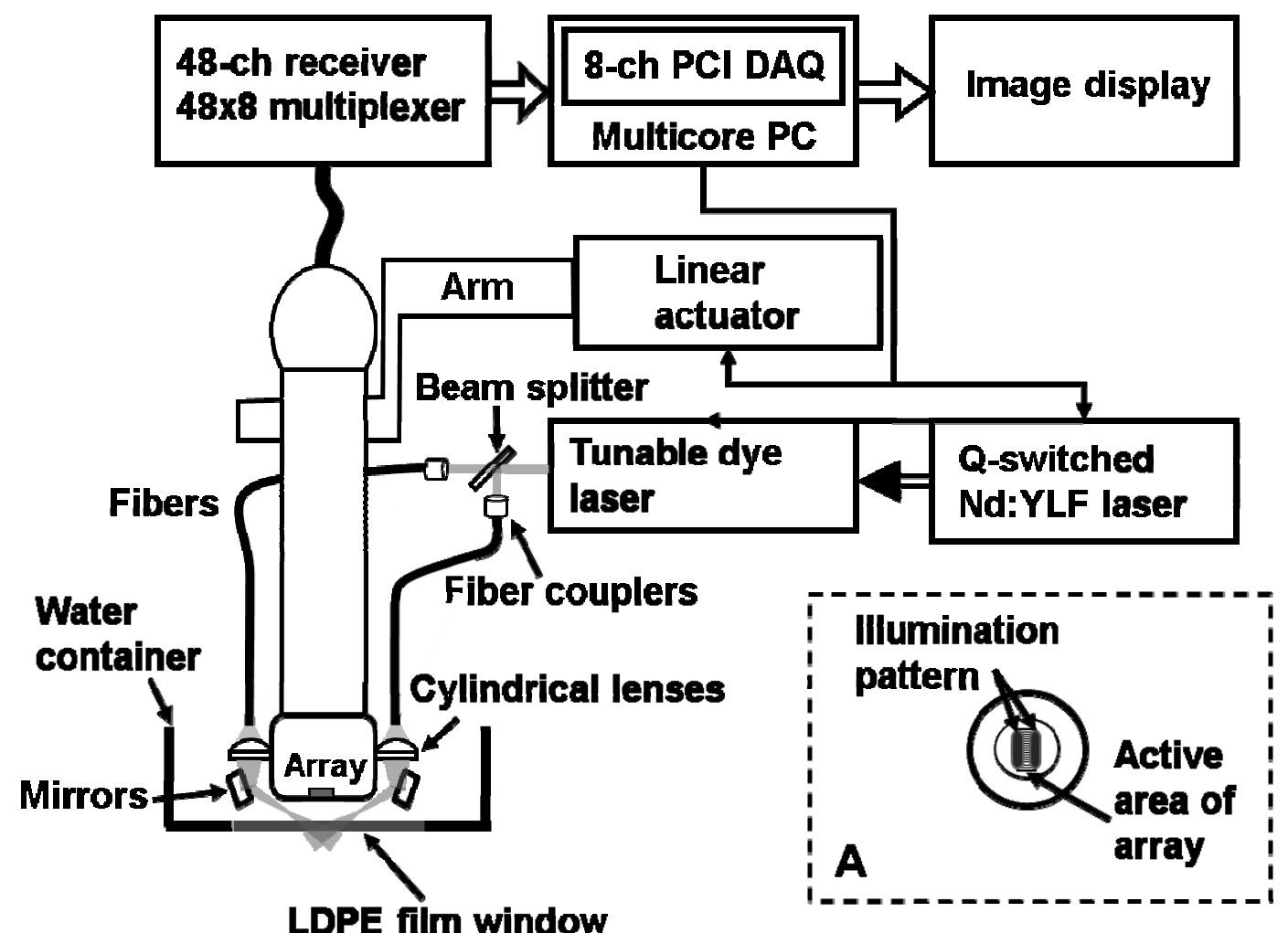

Fig. 1. Schematic of the 3-D dark-field reflection-mode photoacoustic imaging system. Inset A, illumination patterns on the skin surface.

Dark-field laser pulse illumination was achieved through fine tuning the mirrors and cylindrical lenses, reducing the photoacoustic signals from the superficial paraxial area. However, a large dark-field area may reduce the optical fluence being able to reach the targeted area. The optimal illumination radius was estimated to be $7 \mathrm{~mm}$ using the concept of effective attenuation coefficient [15], the exponential decay rate of fluence far from the source, with typical tissue parameters. Consequently, leaving $\sim 1 \mathrm{~mm}$ width right below the array elements as the dark field gives approximately the best performance. In practice, the mirrors and cylindrical lenses were finely tuned to optimize the SNR.

The optical fluence on the skin surface was estimated to be $\sim 1-2 \mathrm{~mJ} / \mathrm{cm}^{2}$ per pulse, well below the ANSI recommended Maximum Permissible Exposure (MPE) of $20 \mathrm{~mJ} / \mathrm{cm}^{2}$ for a single pulse. We acquired data in $1 \mathrm{~s}-50$ frames for realtime B-scan imaging or 166 frames for 3-D imaging; the time average light intensity during this $1 \mathrm{~s}$ was $\sim 600 \mathrm{~mW} / \mathrm{cm}^{2}$ (the total illuminated surface area for one 3-D image was 1.2 $\mathrm{cm}^{2}$ due to the mechanical scanning), also below the ANSI recommended MPE calculated as $1.1 \mathrm{t}^{0.25} \mathrm{~J} / \mathrm{cm}^{2}$ [16]. For prolonged illumination, the ANSI recommended MPE for average light intensity would be lower. However, for prolonged illumination during B-scan imaging, we can either give a break of a few seconds between each acquisition or slow down the frame rate. We also expect to reduce the delivered energy with improved SNR by system optimization. The ANSI safety limit is dominantly based on the thermal mechanism; thus our compliance to the ANSI standards assumes no thermal damage to the tissue. 


\subsection{Ultrasound Array and Beamforming}

We used a unique 30-MHz ultrasound linear array fabricated from a 2-2-piezo-composite by the NIH Resource Center for Medical Ultrasonic Transducer Technology at the University of Southern California [17]. The array had 48 elements (of dimensions $82 \mu \mathrm{m} \times 2 \mathrm{~mm}$ ) with $100-\mu \mathrm{m}$ spacing. The dimension of the element in the elevation direction was $2 \mathrm{~mm}$, and the elements were focused in this direction with a fixed focal length of $8.2 \mathrm{~mm}$, which provides a resolution of $200 \mu \mathrm{m}$ in the elevation direction within the $\sim 3.5 \mathrm{~mm}$ focal zone. The pulse-echo insertion loss and element cross-talk were 19.1 and $-25 \mathrm{~dB}$, respectively. The mean fractional bandwidth was $50 \%$ for pulse-echo operation, which translates to $\sim 70 \%$ for receiving-only operation, as used in our present photoacoustic imaging system.

While ultrasound beamforming traditionally has used dedicated hardware $[18,19]$, we instead used multi-core processors (Dell Precision 490 with two $2.66 \mathrm{GHz}$ Quad core Xeon processors), which allows off-the-shelf personal computers to perform the task and offers programming flexibility. Microsoft Visual Studio 2005 Professional Edition and Microsoft XNA Game Studio in the Visual Studio C\# 2005 Express Edition environment were used to develop the software for dynamic receive beamforming and display. Details on implementation of multi-threaded parallel programming and GPU-based scan conversion and display in this software beamforming can be found elsewhere [12].

\subsection{Data Acquisition and Volume Imaging}

Photoacoustic signals picked up by the ultrasound array were amplified by a custom-built RF board with a $33-73 \mathrm{~dB}$ variable gain and were down-multiplexed to eight channels, which were digitized at 125 mega-samples per second (MSPS) using a 14-bit 8-channel PCI DAQ card (Octopus CompuScope 8389, GaGe Applied Systems, USA). The card was used as the master clock for the entire system and was programmed to send trigger signals to the multiplexer control and laser. The repetition rate was set at $1 \mathrm{kHz}$, which was the highest rate that the laser could work without degradation of pulse energy.

We used linear scanning to achieve 3-D imaging. During the scanning, the array translated linearly over the skin surface, so that the B-scan imaging planes were all parallel to each other. This was accomplished by mounting the light delivery system and the ultrasound array in a linear motion actuator (KR20, THK CO. LTD., Japan). A bipolar stepper motor (4118S, Lin Engineering, USA) controlled by a microstep stepper motor controller (BC2D15, Peter Norberg Consulting, Inc., USA) was used to drive the linear motion actuator. The scanning system provided sufficient precision $(20 \mu \mathrm{m})$ for our use. Six laser shots were needed to obtain one B-scan image because of the 6:1 down-multiplexing in our data acquisition. To produce one 3-D image, $166 \mathrm{~B}$-scan frames were acquired in $996 \mathrm{~ms}$, corresponding to 996 laser shots at a $1-\mathrm{kHz}$ repetition rate. During the data acquisition, the array scanned continuously at a constant speed (10 $\mathrm{mm} / \mathrm{s}$ ). The speed was set so that the distance the array travelled during each B-scan time period was $60 \mu \mathrm{m}$, less than the ultrasonic focus in the elevational direction $(200 \mu \mathrm{m})$. Although the linear motion actuator was fixed on an optical table for scanning, handheld operation was also possible. More user-friendly handheld operation for daily clinical use can be achieved by shrinking the size of the light delivery and scanning systems through custom manufacturing.

While realtime B-scan imaging was demonstrated at 50 frames per second (FPS) [13], postbeamforming after data acquisition reached 83 FPS. This higher speed was due to less hardware communication. In post-beamforming, a B-scan movie was first played, and an MAP of the acquired 3-D image was then displayed for preview immediately. In total, $\sim 2-3 \mathrm{~s}$ was needed for a user to view an MAP image, representing the fastest speed among reported high frequency photoacoustic imaging systems. The user might choose to either replay the B-scan movie or display a contrast-enhanced MAP image processed by a Dynamic-link Library (DLL) written in MATLAB (Math Works, Inc., USA). All these operations could be done online by simply clicking corresponding buttons on a graphic user interface (GUI) generated by the C\# program. 


\section{RESULTS}

The spatial resolution of our system was characterized by imaging 6 - $\mu$ m-diameter carbon fibers in water. The axial, lateral, and elevational resolutions (at $\sim 7 \mathrm{~mm}$ normal depth from the transducer surface) were estimated to be 25,70 , and $200 \mu \mathrm{m}$, respectively. The imaging depth was demonstrated to be greater than $3 \mathrm{~mm}$ in scattering biological tissue.

To demonstrate the system's capability to image blood vessels in vivo, we imaged the upper dorsal region of a Sprague Dawley rat (Harlan Sprague Dawley, Inc., USA), $280 \mathrm{~g}$ in weight. The rat was anesthetized by intramuscular injection of a mixture of Ketamine $(85 \mathrm{mg} / \mathrm{kg})$ and Xylazine $(15 \mathrm{mg} / \mathrm{kg})$. The hair in the imaged region was removed with commercial hair-removal lotion before imaging. Acoustic coupling gel was applied to improve acoustic coupling between the animal and the LDPE film. After the experiment, the rat recovered normally, without noticeable health problems. All experimental animal procedures were carried out in compliance with approved protocols.
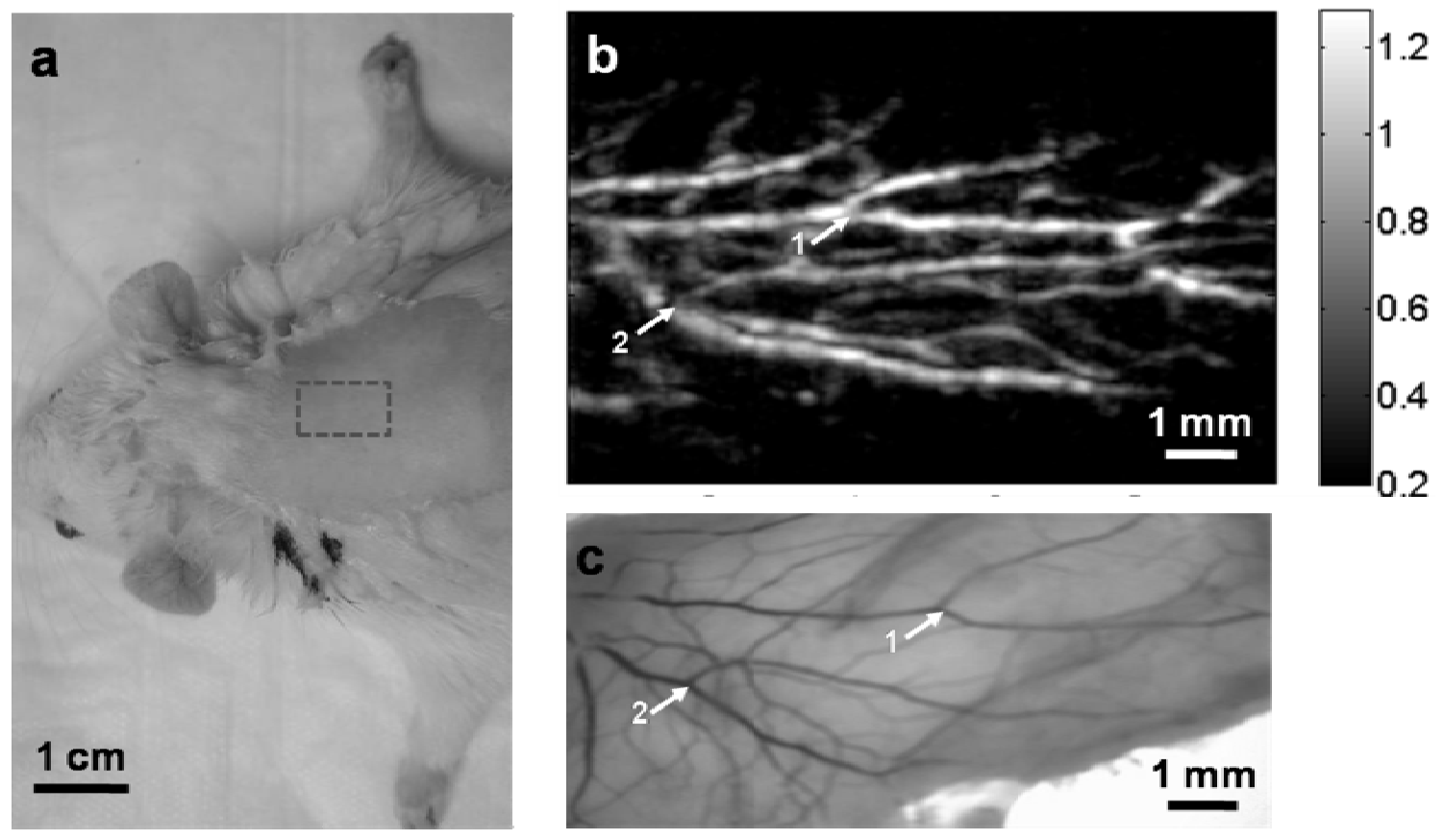

Fig. 2. (a) Photograph of the Sprague Dawley rat with hair removed before photoacoustic imaging; the rectangle in dash lines indicates the imaged area. (b) In vivo noninvasive maximum amplitude projection (MAP) photoacoustic image. The grayscale represents relative optical absorption (arbitrary unit). (c) Ex vivo invasive optical microscopic image taken from the dermal side of the excised skin. Numbers 1 and 2 indicate the corresponding vessels in the MAP and microscopic images.

Fig. 2 shows an in vivo noninvasive MAP photoacoustic image and an ex vivo invasive transmission optical microscopic image of the subcutaneous vasculature in an imaged area. The photoacoustic image is a gray-level plot of the maximum amplitude of the envelope of each time-resolved (A-scan) photoacoustic signal. The transmission optical microscopic photograph $(\sim 4 \times 10 \mathrm{~mm})$, partially overlapping with the photoacoustic image, was acquired ex vivo from the dermal side of the excised skin. As shown in Fig. 2, the 
vascular distributions agreed well between the two images. Vessels shown in the photoacoustic image had diameters varying from $\sim 70$ to $300 \mu \mathrm{m}$. The data acquisition of the photoacoustic image was completed within $1 \mathrm{~s}$, and the MAP image was displayed within $2-3 \mathrm{~s}$. Fig. 3 shows the corresponding vessels in the MAP image and a typical B-scan image. It can be seen that the imaged vasculature consists of subcutaneous blood vessels within $1-2 \mathrm{~mm}$ depth below the skin surface.
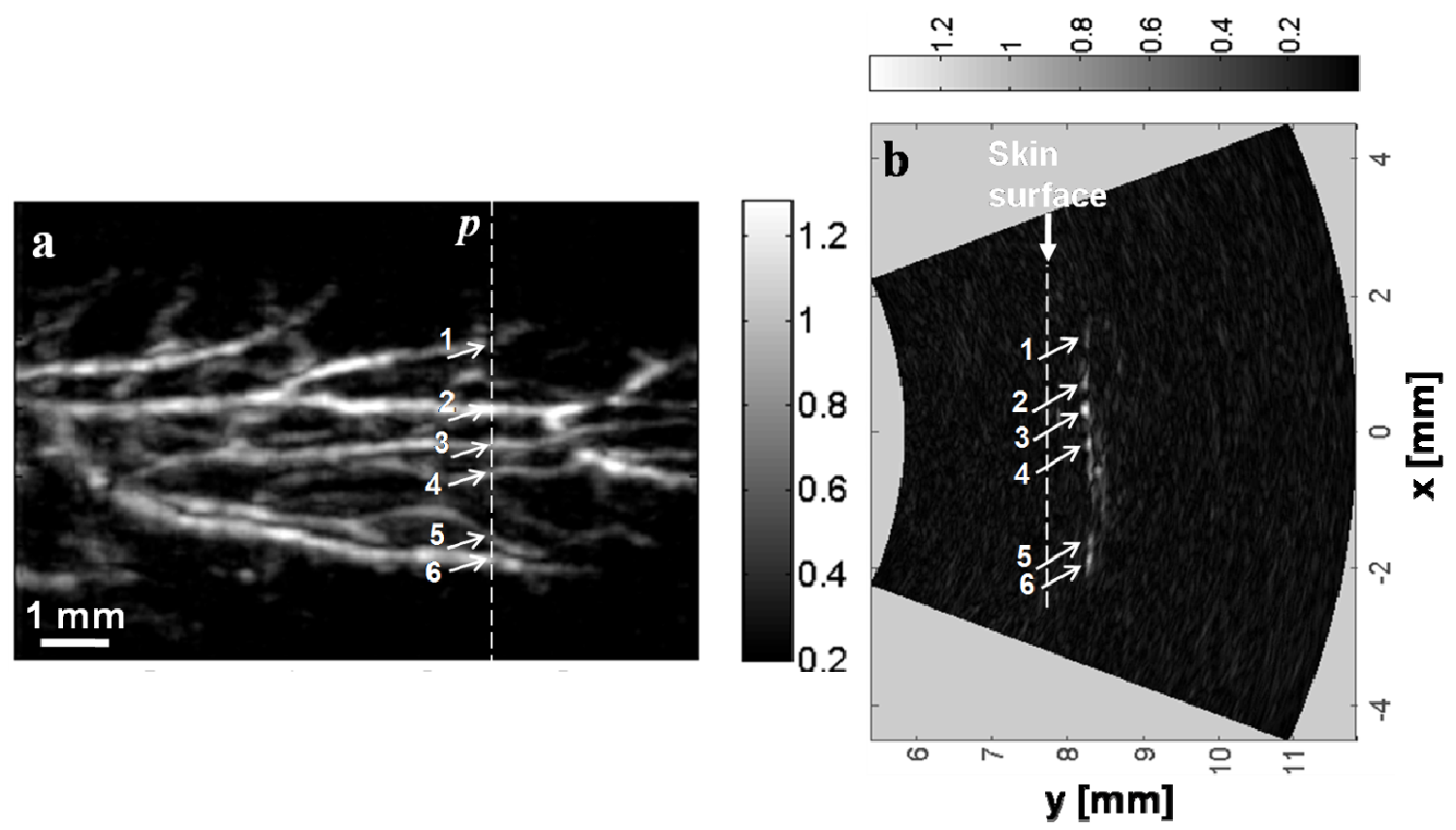

Fig. 3. (a) Maximum amplitude projection (MAP) photoacoustic image. (b) B-scan image at position $P$ in a; $\mathrm{x}$ and $\mathrm{y}$ denote the array direction and the depth direction, respectively. Numbers $1-6$ indicate the corresponding vessels in the MAP and B-scan images.

A 3-D image obtained from post-processing using VolView (Kitware Inc., USA) is shown in Fig. 4. Interactive features such as rotation and scaling were available in VolView, which enable visualization from different perspectives and detailed analysis on the regions of interests. 


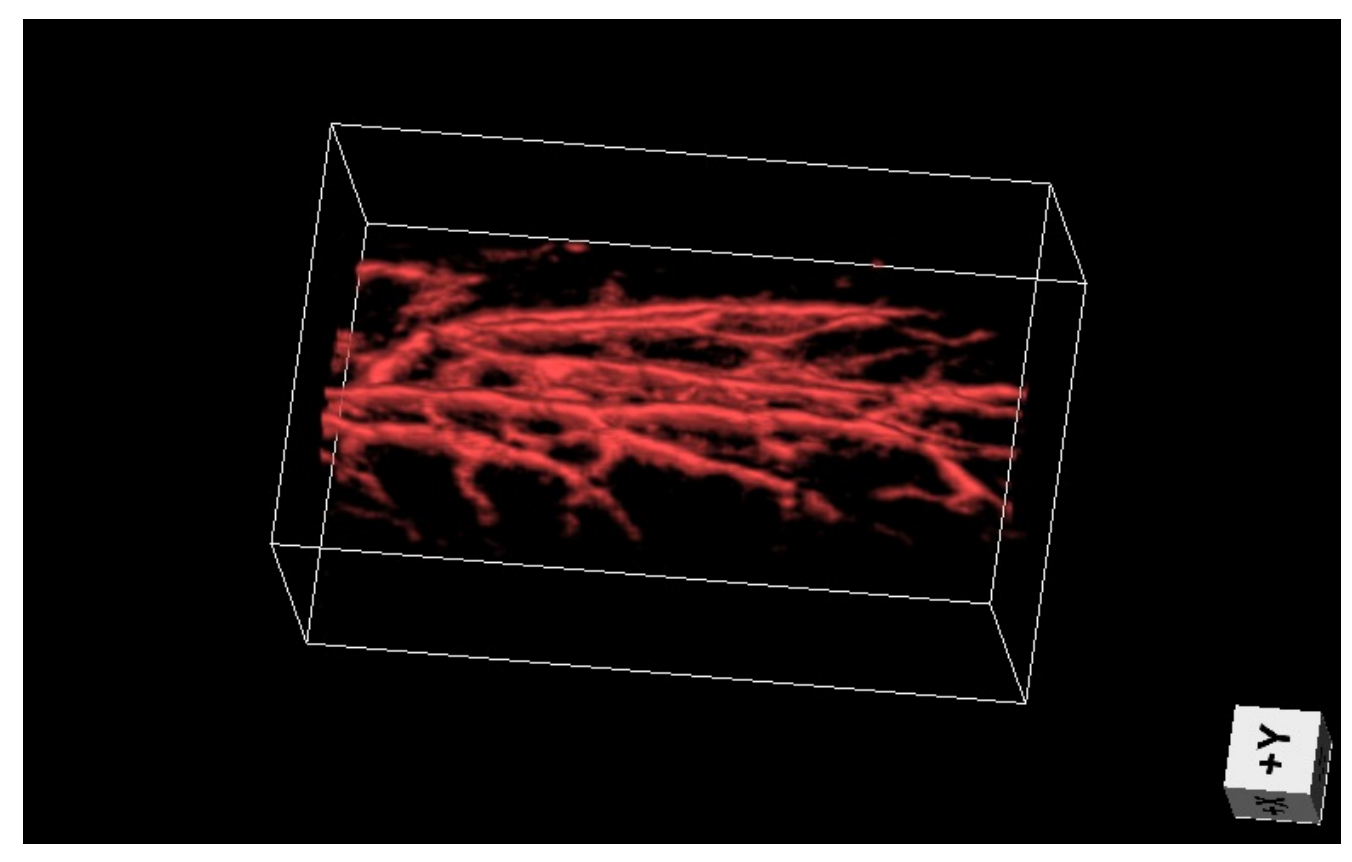

Fig. 4. Three-dimensional visualization of the in vivo photoacoustic image.

\section{CONCLUSIONS AND DISCUSSION}

We have demonstrated fast 3-D photoacoustic microscopy with a $30-\mathrm{MHz}$ ultrasound linear array capable of realtime B-scan imaging at $50 \mathrm{~Hz}$ and 3-D imaging at $1 \mathrm{~Hz}$. To our knowledge, this is the fastest high frequency photoacoustic imaging achieved hitherto. The system integrates a novel light delivery system and a motorized linear motion actuator with the ultrasound array, performing scans in elevational direction for 3-D imaging. The light delivery system enables robust reflection-mode imaging, which is applicable to more anatomical sites than orthogonal- or transmission-mode imaging. Optical dark-field illumination is also achieved, reducing the otherwise strong interference of the extraneous photoacoustic signals from the superficial paraxial area.

Previous studies have demonstrated that photoacoustic imaging has great potential in early cancer diagnosis, blood oxygenation mapping, and functional brain imaging. Because of the unique realtime 2D and fast 3-D imaging capability, our system is anticipated to facilitate many of these applications. The compact system design also favors clinical practices. Particularly, the promising results shown in this report should motivate future development of photoacoustic microscopy with high frequency ultrasound arrays towards clinical applications.

\section{ACKNOWLEDGEMENTS}

This work was sponsored by grants R01 EB000712, R01 EB008085, R01 NS46214, and U54 CA136398 (Network for Translational Research) from the National Institutes of Health. L.W. has a financial interest in Endra, Inc., which, however, did not support this work. 


\section{REFERENCES}

1. L. V. Wang and H. I. Wu, Biomedical optics: principles and imaging, John Wiley \& Sons, Hoboken, New Jersey (2007).

2. H. F. Zhang, K. Maslov, G. Stoica, and L. V. Wang, "Functional photoacoustic microscopy for highresolution and noninvasive in vivo imaging," Nat. Biotechnol. 24, 848-851 (2006).

3. J. T. Oh, M. Li, H. F. Zhang, K. Maslov, G. Stoica, and L. V. Wang, "Three-dimensional imaging of skin melanoma in vivo by dual-wavelength photoacoustic microscopy," J. Biomed. Opt. 11, 034032 (2006).

4. R. M. Weight, J. A. Viator, P. S. Dale, C. W. Caldwell, and A. E. Lisle, "Photoacoustic detection of metastatic melanoma cells in the human circulatory system," Opt. Lett. 31, 2998-3000 (2006).

5. S. Manohar, S. E. Vaartjes, J. C. G. van Hespen, J. M. Klaase, F. M. van den Engh, W. Steenbergen, and T. G. van Leeuwen, "Initial results of in vivo non-invasive cancer imaging in the human breast using nearinfrared photoacoustics," Opt. Express 15, 12277-12285 (2007).

6. S. A. Ermilov, A. Conjusteau, K. Mehta, R. Lacewell, P. M. Henrichs, and A. A. Oraevsky, "128channel laser optoacoustic imaging system (LOIS-128) for breast cancer diagnostics," Proc. SPIE, 6086, 68-79 (2006).

7. X. Wang, Y. Pang, G. Ku, X. Xie, G. Stoica, and L. V. Wang, "Non-invasive laser-induced photoacoustic tomography for structural and functional imaging of the brain in vivo," Nat. Biotechnol. 21, 803-806 (2003).

8. S. Yang, D. Xing, Y. Lao, D. Yang, L. Zeng, L. Xiang, and W. R. Chen, "Noninvasive monitoring of traumatic brain injury and post-traumatic rehabilitation with laser-induced photoacoustic imaging," Appl. Phys. Lett. 90, 243902 (2007).

9. M. Li, J. Oh, X. Xie, G. Ku, W. Wang, C. Li, G. Lungu, G. Stoica, and L. V. Wang, "Simultaneous molecular and hypoxia imaging of brain tumors in vivo using spectroscopic photoacoustic tomography," Proceedings of IEEE 96, 481-489 (2008).

10. J. Niederhauser, M. Jaeger, R. Lemor, P. Weber, and M. Frenz, "Combined ultrasound and optoacoustic system for real-time high contrast vascular imaging in vivo," IEEE Trans. Med. Imaging 24, 436-440 (2005).

11. D. W. Yang, D. Xing, S. H. Yang, and L. Z. Xiang, "Fast full-view photoacoustic imaging by combined scanning with a linear transducer array," Opt. Express 15, 15566-15575 (2007).

12. R. Zemp, M. Li, R. Bitton, K. K. Shung, G. Stoica, and L. V. Wang, "Photoacoustic imaging of the microvasculature with a high-frequency ultrasound array transducer," J. Biomed. Opt. 12, 010501 (2007).

13. R. J. Zemp, L. Song, R. Bitton, K. K. Shung, and L. V. Wang, "Realtime photoacoustic microscopy in vivo with a 30-MHz ultrasound array transducer," Opt. Express 16, 7915 (2008).

14. R. J. Zemp, L. Song, R. Bitton, K. K. Shung, and L. V. Wang, "Realtime photoacoustic microscopy of murine cardiovascular dynamics," Opt. Express 16, 18551 (2008).

15. L. V. Wang, W. R. Chen, and R. E. Nordquist, "Optimal beam size for light delivery to absorptionenhanced tumors buried in biological tissue and effect of multiple beam delivery: a Monte Carlo study," Appl. Opt. 36, 8286-8291 (1997). 
16. The Laser Institute of America, American National Standard for Safe Use of Lasers (ANSI Z136.12000), The Laser Institute of America (2000).

17. J. M. Cannata, J. A. Williams, Q. F. Zhou, T. A. Ritter, and K. K. Shung, "Development of a 35-MHz piezo-composite ultrasound array for medical imaging," IEEE Trans. Ultrason. Ferroelectr. Freq. Control 53, 224-236 (2006).

18. K. Wall and G. R. Lockwood, "Modern implementation of a realtime 3-D beamformer and scan converter system," IEEE Ultrasonics Symposium, 1400-1403 (2005).

19. C. H. Hu, X. C. Xu, J. M. Cannata, J. T. Yen, and K. K. Shung, "Development of a real-time, highfrequency ultrasound digital beamformer for high-frequency linear array transducers," IEEE Trans. Ultrason. Ferroelectr. Freq. Control 53, 317-323 (2006). 